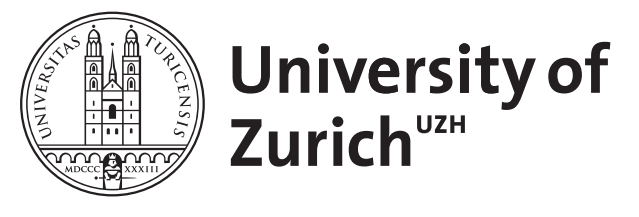

\title{
Neue Konzepte und Substanzen zur Radiosensibilisierung
}

Pruschy, M

\begin{abstract}
Die Entwicklung neuer Therapieformen im Bereich der kombinierten Radiochemotherapie ist ein wichtiger Bestandteil der Grundlagenforschung und angewandten Radiobiologieforschung. Ionisierende Strahlung schädigt nicht nur die DNA, sondern induziert ein breites Spektrum an Stressantworten in der bestrahlten Zelle und im Tumor, die unabhängig von der eigentlichen DNA-Schädigung auftreten. Nun stellen sowohl Signalkaskaden, die von der DNA-Schädigung ausgehen, als auch biologische Prozesse, die entfernt von der DNA durch ionisierende Strahlung induziert werden, neue Zielstrukturen für hoch spezifische Substanzen dar, um die Zelle für die ionisierende Strahlung zu sensibilisieren. In diesem Übersichtsartikel werden neue Konzepte anhand der klassischen "4 R" der Radiobiologie vorgestellt und diskutiert
\end{abstract}

DOI: https://doi.org/10.1007/s00761-007-1187-y

Posted at the Zurich Open Repository and Archive, University of Zurich ZORA URL: https://doi.org/10.5167/uzh-155910

Journal Article

Published Version

Originally published at:

Pruschy, M (2007). Neue Konzepte und Substanzen zur Radiosensibilisierung. Der Onkologe, 13(3):263268.

DOI: https://doi.org/10.1007/s00761-007-1187-y 
Onkologe 2007 · 13:263-268

DOI 10.1007/s00761-007-1187-y

Online publiziert: 28. Februar 2007

(c) Springer Medizin Verlag 2007
M. Pruschy

Labor für Molekulare Radiobiologie, Klinik für Radio-Onkologie,

Universitätsspital Zürich

\section{Neue Konzepte und Substanzen zur Radiosensibilisierung}

Die Identifikation von neuen Kombinationsmodalitäten im Bereich der Radiochemotherapie braucht ein besseres Verständnis der direkten Wirkung ionisierender Strahlung auf den Tumor. Dadurch können neue Zielstrukturen definiert werden, die sich für die Kombination mit hoch wirksamen, spezifischen und klinisch relevanten Substanzen anbieten. Mechanistik- und effizienzorientierte Forschung in diesem Bereich haben während den letzten Jahren zu grundlegenden neuen Konzepten beigetragen. Hier steht nicht mehr die Erhöhung des Schadens in der Zelle durch die ionisierende Strahlung in Kombination mit Zytotoxika im Vordergrund, sondern die Steigerung der Effizienz des induzierten Schadens, der letztlich zum Absterben des Tumors führen soll.

Die klinische Radiotherapie hat sich während den letzten 100 Jahren von einer experimentellen Anwendung der Röntgenstrahlung zu einer komplexen, technologisch höchst anspruchsvollen und breit angewandten Therapiemodalität verändert. Zurzeit werden mehr als 50\% aller Patienten mit einem soliden Tumor entweder mit Radiotherapie alleine oder im Rahmen einer kombinierten Therapie mit Chirurgie und/oder Chemotherapie behandelt. Die wohl wichtigsten technologischen Fortschritte, welche die Entwicklung der Radiotherapie prägten, waren die Einführung von CT und MRI, welche die genau 3D-Lokalisation des Tumors ermöglichten, und die Weiterentwicklung der Linearbeschleunigertechnologie, die heutzutage die präzise Bestrahlung des eigentlichen Tumors mit gleichzeitiger Reduktion des Bestrahlungsvolumens und somit Schonung des gesunden Gewebes erlauben. Diese Entwicklung hat zu der heute eingesetzten IMRT-Technologie (,intensity-modulated radiation therapy") in der klinischen Radiotherapie geführt. Trotz dieser technischen Fortschritte wird jedoch eine unlimitierte Dosiseskalation ohne weitere größere Normalgewebstoxizitäten auch in der Zukunft kaum möglich sein $[2,3,5]$.

Parallel zu diesen technologischen Errungenschaften machte die Radioonkologieforschung enorme Fortschritte im Bereich des biologischen Verständnisses der Strahlenwirkung. Wir verstehen heutzutage die Strahlenwirkung nicht nur auf der Ebene der DNA-Schädigung, sondern erkennen, dass die unterschiedliche Strahlensensitivität von verschiedenen Tumorund Normalgewebszellen auch auf ein komplexes Netzwerk von intra- und interzellulären Signalübermittlungskaskaden zurückzuführen ist. Diese Erkenntnisse haben zur Entwicklung von neuen Rationalen geführt, wie eine Strahlenresistenz als Teil einer kombinierten Radiochemotherapie mit Radiosensibilisatoren reduziert werden kann. Dabei sollen kleinmolekulare Substanzen spezifische Signalübermittlungskasdaden so modulieren, dass die Tumorzelle durch die Strahlung sensibilisiert, aber das Normalgewebe nicht weiter beeinträchtigt und dadurch die therapeutische Breite erhöht wird (• Abb. 1).
Die klassische Radiobiologie hat während den letzten 100 Jahren die Antwort der Zelle bzw. des Tumors und des Normalgewebes auf die Bestrahlung in 4 bzw. 5 Kategorien eingeteilt, welche „die 4-5 R der Radiotherapie " genannt werden (Repair, Redistribution/Reassortment, Repopulation und Reoxygenation). Diese Einteilung basiert auf der jahrzentelangen Charakterisierung der Gewebsreaktion auf die Bestrahlung, ohne die genauen molekularen und zellulären Zusammenhänge genau verstanden zu haben. Die moderne Radiobiologie kann heute diese Kategorien und somit die Antwort auf die ionisierende Strahlung auf molekularer Ebene untersuchen. Nun bieten diese Kategorien heute eine Möglichkeit, die enorme Fülle von molekularen Prozessen zu ordnen, und können als Leitfaden dienen, neue Zielstrukturen für eine kombinierte Radiochemotherapie mit kleinmolekularen Substanzen und Signalübermittlungsinhibitoren zu definieren. Im Folgenden werden neueste Entwicklungen im Bereich der kombinierten Radiochemotherapie anhand dieser klassischen Kategorien vorgestellt $([4,10] ;$; Abb. 2).

\section{Reparatur der DNA als Zielstruktur für kombinierte Radiochemotherapie}

Ionisierende Strahlung induziert verschiedene Arten von DNA-Schäden. DNADoppelstrangbrüche (DSB) stellen dabei bezüglich genomischer Integrität und Zellüberleben nach Bestrahlung die gravierendsten Veränderungen des Genoms 
dar und können durch zwei unterschiedliche DNA-Reparaturmechanismen repariert werden:

- Nicht-homologes-End-Joining

(NHEJ) und

- homologe Rekombination (HR).

Die molekularen Bestandteile und die Funktion beider Reparaturmaschinerien konnten während der letzten Jahre im Detail aufgeschlüsselt werden. Während NHEJ ein fehleranfälliges DSB-ReparaturSystem ist und über den ganzen Zellzyklus hinweg aktiv ist, ist HR nur während der S-Phase und der G2-Phase des Zellzyklus aktiv, funktioniert aber unter Verwendung des intakten Schwesterchromatids bzw. des 2. homologen, intakten Chromosoms fehlerfrei. Dass sich diese Reparaturmaschinerien als Zielstruktur zur Radiosensibilisierung eignen, wurde mit verschiedenen, experimentellen Systemen geprüft. So zeigen z. B. Zelllinien von Patienten mit spezifischen Mutationen (z. B. ATM, NBS) oder Zellen, welche durch künstliches Ausschalten von Genprodukten mittels siRNA-Interferenz manipuliert wurden, im Unterschied zu Zellen mit intaktem NHEJ oder HR eine deutlich erhöhte Radiosensitivität [19, 26].

Verschiedene Substanzen wurden bereits in vitro und in vivo getestet, welche die DNA-Proteinkinase (DNA-PKc) inhibieren. DNA-PKc gehört zur Familie der PI-3-Kinasen und ist ein Schlüsselenzym des NHEJ. Der irreversible Hemmstoff Wortmannin und der ATP-kompetitive Inhibitor LY294002 stellen dabei die bekanntesten Vertreter der NHEJInhibitoren dar. Beide Substanzen sensibilisieren Tumorzellen für die Behandlung mit ionisierender Strahlung. Da beide Substanzen jedoch zu wenig selektiv sind und auch weitere $\mathrm{PI}_{3}$-Kinasen-Klassen hemmen, die in der Zellzyklusregulation und antiapoptotischen Signalübermittlungskaskaden eine wichtige Funktion spielen, können sie vorwiegend nur für In-vitro-Experimente eingesetzt werden. Anhand von Strukturaktivitätsstudien über LY294002 konnten unterdessen verschiedene DNA-PKc-inhibitoren entwickelt werden (NU7026 und NU7441), die selektiver wirken, einen hohen DosisModifikationsfaktor in Kombination mit Bestrahlung aufweisen (1,3 bzw. 2,5) und in präklinischen In-vivo-Versuchen eingesetzt werden können. Screenings von kleinmolekularen Substanzbibliotheken mit anschließender Struktur-Funktionsanalyse und Derivatisierung lieferten ebenfalls Erfolg versprechende selektive DNA-PKc-Hemmstoffe. Sowohl IC86621 als auch das Indolderivat SU11752 hemmen NHEJ, werden bereits in Kombination mit Bestrahlung in vivo ausgetestet und bestätigen, dass DNA-PKc eine attraktive Zielstruktur zur Radiosensibilisierung auf der Ebene der DNA-Reparatur ist $[11,15,17]$.

Aufgrund der erhöhten Aktivität der HR während der S- und G2-Phase des Zellzyklus versprechen Inhibitoren der HR ein breiteres therapeutisches Fenster. Leider gibt es jedoch noch keine hochspezifischen direkten Hemmstoffe. Künstliche Zellsysteme oder Zellen versetzt mit siRNA bzw. Antisens-Konstrukten (z. B. gegen ATM) zeigen eine deutlich erhöhte Radiosensitivität. Parallel zeigten Versuche mit dem klinisch relevanten Inhibitor Gleevec (STI-571), welches eine spezifische Reduktion der HR-Proteines Rad51 induziert, dass Inhibition von HR auch von klinischem Interesse sein kann [23].

Auch wenn im Bereich der kombinierten Radiochemotherapie mit Inhibitoren der DNA-Reparatursysteme präklinische Daten sehr Erfolg versprechend aussehen und in Zukunft weitere Zielstrukturen dieser Reparatursysteme identifiziert und mit noch spezifischeren und selektiveren Substanzen inhibiert werden können, darf die Gefahr einer erhöhten Karzinogenese durch solch eine Kombination nicht außer Acht gelassen werden. Dennoch besteht zurzeit ein reges Interesse im Bereich der angewandten Radiobiologie die Möglichkeiten solcher Kombinationsmodalitäten weiterzuentwickeln und auszutesten.

\section{Redistribution als Zielstruktur für die kombinierte Radiochemotherapie}

Zellzyklusabhängige Unterschiede in der Strahlensensitivität von Tumorzellen und Normalgewebszellen wurden ursprünglich durch Synchronisationsexperimente an Zellkulturen identifiziert. Eine deutlich erhöhte Radiosensitivität kurz vor der

\section{Hier steht eine Anzeige.}

Springer 
Mitose ist durch die nichtreparierte Verschleppung von DNA-Schäden durch die Mitose erklärbar, sodass eine mitotische Katastrophe bzw. genetische Instabilität in den Tochterzellen zum Zelltod führt. Eine erhöhte Resistenz ist in der späten S-Phase erkennbar, dies wohl aufgrund gesteigerter DNA-Reparaturkapazitäten in der Zelle. Wegen des genauen molekularen Verständnisses der Zellzyklusregulation nach Stress oder z. B. nach Bestrahlung gewinnen heute Elemente der sog. Zelllzyklus-Checkpoints für die Radiosensibilisierung an Bedeutung. Eine weitere Möglichkeit stellt die pharmakologische Redistribution der zu bestrahlenden Zellen in eine radiosensitive Phase dar. Verschiedene klinische Strategien wurden hierzu entwickelt, die jedoch meistens aufgrund der Heterogenität eines Tumors scheiterten. Es muss jedoch betont werden, dass ein klassisches Fraktionierungsschema einer Radiotherapie ebenfalls eine Redistribution und Zellzyklussynchronisation durch strahleninduzierte, vorübergehende Zellzyklusstopps bewirkt und dieser Mechanismus ebenfalls zum Erfolg einer fraktionierten Radiotherapie beisteuert [18, 22, 27].

Als Beispiel der Wirkungsweise einer Substanz, die einen spezifischen Zellzykluscheckpoint außer Kraft setzen kann und dadurch radiosensibilisierend wirkt, dient das 7-Hydroxystaurosporinederivat UCN-o1, das die Checkpoint Kinase Chk1 hemmt. Zellen reagieren auf die Bestrahlung mit einer Aktivierung eines G2-Zellzyklusstopps, der durch eine Chk1-abhängige Signalübermittlungskaskade induziert wird. Blockierung dieser Kinase durch UNC-o1 unterbindet diesen Zellzyklusstopp und den Zellen wird keine Möglichkeit geboten, die strahleninduzierten Schäden vor der Zellteilung zu reparieren. Dies führt zu einem Anstieg an chromosomalen Schäden und vermehrtem Zelltod. Im Unterschied zu Tumorzellen, die oftmals keinen intakten G1-Checkpoint mehr besitzen und dadurch umso mehr auf einen intakten G2-Checkpoint angewiesen sind, arretieren proliferierende Normalgewebszellen nach einem Strahleninsult zu einem großen Teil bereits vor der DNA-Synthese (G1-Checkpoint) und werden somit durch die UCN-o1- Behandlung kaum beeinträchtigt. Zelluläre In-vitro-Versuche be- stätigten diesen Wirkmechanismus von UCN-O1 und ein ausgeprägter Radiosensibilisierungsfaktor konnte bestimmt werden. In-vivo-Experimente mit verschiedenen Tumorsystemen zeigten jedoch keinen Erfolg. Interessanterweise zeigen weitere Substanzklassen, die noch selektiver Chk1 inhibieren (SB218078 und CEP-3891) ein ähnliches Wirkungsspektrum wie UCN-o1 und lassen die Frage offen, ob gezielte Inhibierung eines Zellzykluscheckpoints von klinischem Interesse sein wird $[16,25]$.

Im Unterschied $\mathrm{zu}$ dieser Strategie können chromosomale Aberrationen auch vermehrt durch die forcierte Akkumulation von Zellen in die End-G2-Phase bzw. M-Phase des Zellzyklus und anschließende Bestrahlung bewirkt werden. Hiezu können Mikrotubuli stabilisierende Substanzen wie z. B. Taxane oder Epothilone verwendet werden. Diese Substanzklassen sind des Weiteren von Interesse, da sie zusätzlich zu ihrem Zellzykluseffekt auch Apoptose in p53-negativen, strahlen- und chemotherapieresistenten Tumorzellen induzieren. Im Unterschied zu Taxanen, deren Einsatz oftmals durch hohe Nebenwirkungen limitiert ist, zeichnen sich Epothilone durch eine bessere Verträglichkeit und keine MultidrugResistenz aus. Beide Substanzklassen zeigen präklinisch in vitro und in vivo eine radiosensibilisierende Wirkung und werden klinisch getestet [13]. Auch wenn diese Strategien mit spezifischen Substanzen klinisch noch nicht etabliert sind, ist die Modulation des Zellzyklus zur Radiosensibilisierung von Tumorzellen konzeptionell sehr viel versprechend.

\section{Reoxygenierung und Tumorangiogenese als Zielstruktur für die kombinierte Radiochemotherapie}

Da normoxische Tumorzellen strahlensensibler sind, steigt die relative hypoxische Fraktion innerhalb des Tumors direkt nach Bestrahlung mit einer hohen Einzeldosis. Die daran anschließende langsame Reduktion des Anteils hypoxischer Zellen im Tumors wird unter Reoxygenierung im klassischen radiobiologischen Sinne verstanden. Ein fraktioniertes Bestrahlungsschema in der Klinik greift auch bei
Onkologe 2007 · 13:263-268

DOI 10.1007/s00761-007-1187-y

(c) Springer Medizin Verlag 2007

\section{Pruschy \\ Neue Konzepte und Substanzen zur Radiosensibilisierung}

\section{Zusammenfassung}

Die Entwicklung neuer Therapieformen im Bereich der kombinierten Radiochemotherapie ist ein wichtiger Bestandteil der Grundlagenforschung und angewandten Radiobiologieforschung. lonisierende Strahlung schädigt nicht nur die DNA, sondern induziert ein breites Spektrum an Stressantworten in der bestrahlten Zelle und im Tumor, die unabhängig von der eigentlichen DNA-Schädigung auftreten. Nun stellen sowohl Signalkaskaden, die von der DNA-Schädigung ausgehen, als auch biologische Prozesse, die entfernt von der DNA durch ionisierende Strahlung induziert werden, neue Zielstrukturen für hoch spezifische Substanzen dar, um die Zelle für die ionisierende Strahlung zu sensibilisieren. In diesem Übersichtsartikel werden neue Konzepte anhand der klassischen ${ }^{4} 4 \mathrm{R}^{\prime \prime}$ der Radiobiologie vorgestellt und diskutiert.

Schlüsselwörter

Radioresistenz · DNA-Reparatur · Repopulation · Redistribution · Reoxygenierung

\section{New concepts and substances} for radiosensitization

\section{Abstract}

A tremendous effort in basic and translational radiobiological research exists to identify new treatment modalities using ionizing radiation in combination with novel anticancer agents. lonizing radiation not only damages DNA, but also affects multiple cellular components that induce a multi-layered stress response. These treatment responses can be restricted to the individual cell level but could also be part of an intercellular stress communication network. Both DNA damage induced signaling (which results in cell cycle arrest and the induction of the DNA repair machinery) and also ionizing radiation induced signal transduction cascades, which are generated at cellular sites distant from and independent of DNA damage, represent interesting targets for anticancer treatment modalities for sensitization to ionizing radiation. In this review, we describe current approaches for sensitizing tumor cells with novel anticancer agents along the lines of the classical 4 R's of radiobiology.

Keywords

Radioresistance - DNA repair - Repopulation . Redistribution - Reoxygenation 


\section{Leitthema}

Anti-Signaling Agents

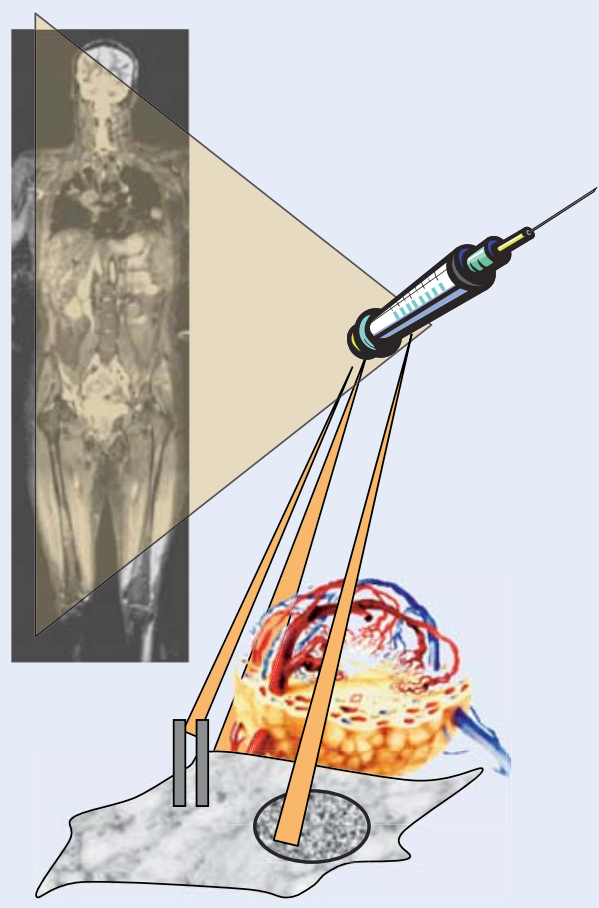

Ionisierende Strahlen

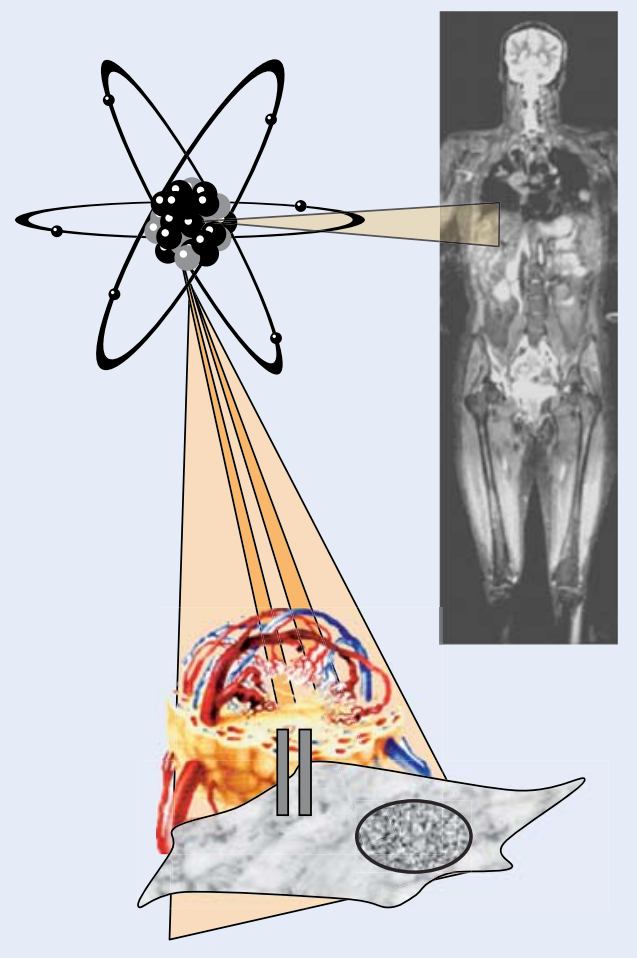

Abb. $1<$ Kombinierte Therapie mit ionisierender Strahlung und , anticancer agents": Auf der Makroebene kann die Bestrahlung lokal appliziert werden, während die Chemotherapie systemisch wirkt. Auf der zellulären Mikroebene wirken die kleinmolekularen Substanzen sehr spezifisch, während die Bestrahlung viele verschiedenste Prozesse induziert (s. Text für Details) tieferen Einzeldosen auf solche Mechanismen zurück, um durch die damit einhergehende repetitive Reoxygenierung und Sensibilisierung den Tumor besser kontrollieren zu können. Während den letzten Jahren zeigte sich, dass Tumoroxygenierung und die Ausbildung einer Tumorhypoxie ein sehr komplexer und multifaktorieller Prozess ist. Der Sauerstoffpartialdruck im Tumor steht in Abhängigkeit einer Balance von Zufuhr und Verbrauch von Sauerstoff und ist im Tumor sehr heterogen. Gleichzeitig muss zwischen einer diffusionsabhängigen chronischen Hypoxie und einer perfusionsabhängigen akuten Hypoxie, induziert durch ungenügend-ausgebildete Tumorangiogenese bzw. Sauerstoffversorgung, unterschieden werden. Der Sauerstoffverbrauch andererseits wird durch den Tumorzellmetabolismus und den Proliferationsstatus mitbestimmt $[7,20]$.

Aufgrund des klassischen, radiobiologischen Dogmas, dass hypoxische Zellen radioresistenter als normoxische Zellen sind, wurde eine kombinierte Radiochemotherapie mit Inhibitoren der Angiogenese lange nicht in Betracht gezogen dies aufgrund der Annahme, dass Inhibierung der Angiogenese zu einer weiteren
Senkung des Sauerstoffpartialdrucks im Tumor führt. Interessanterweise zeigen nun aber präklinische Versuche mit verschiedensten Tumormodellen und Klassen von Antiangiogenetika, dass die Therapieform von ionisierender Strahlung in Kombination mit Antiangiogenetika sehr viel versprechend ist.

Erste Studien in 1995 mit dem Angiogeneseinhibitor TNP-40 zeigten eine verbesserte Tumoroxygenierung und ein verbessertes Ansprechen in Kombination mit Bestrahlung. Diese kombinierten Studien wurden fortgesetzt mit Angiostatin, neutralisierenden Antikörpern gegen VEGF165 und den VEGF-Rezeptor sowie Inhibitoren der VEGF-Rezeptortyrosinkinase-Aktivität (PTK787/ZK222584, SU5416), die alle eine verstärkte Tumorwachstumsverzögerung bewirkten. Zurzeit werden viele präklinische Untersuchungen mit Rezeptortyrosinkinaseinhibitoren in Kombination mit Bestrahlung durchgeführt, die gleichzeitig und doch selektiv, mehrere Tyrosinkinaseaktivitäten blockieren können, z. B. SU11657 (VEGFR2, PDGFR, c-kit), ZD6474, AEE788 (VEGFR2, EGFR). Dies basiert auf der Rationale eines kooperativen Multitarget-Mechanismus. Inhibitoren der Angio- genese können direkt gegen die mikrovaskulären, endothelialen Strukturen gerichtet sein; indirekt kann die Angiogenese aber auch durch die unterdrückte Expression von proangiogenetischen Wachstumsfaktoren in den Tumorzellen gesteuert werden. Ein gleichzeitiges Targeting kann eine verstärkte antiangiogenetische Wirkung induzieren und kann zusätzlich die Tumorzellproliferation reduzieren. Die Regulation dieser Prozesse auf verschiedenen Ebenen gewinnt durch die Kombination mit ionisierender Strahlung weiter an Komplexität, da die Radiotherapie ebenfalls mehrere Gewebsstrukturen im Tumor gleichzeitig bestrahlt [12, 21, 24].

In erster Linie muss erfasst werden, unter welchen Bedingungen (Tumorentität, Klasse von Angiogeneseinhibitor, Kombinationsschema etc.) sich der Tumorhypoxiestatus verändert. Doch wie zu erwarten war, zeigen erste präklinische Versuche unterschiedliche Resultate. Eigene Resultate mit dem Rezeptortyrosinkinaseinhibitor PTK787/ZK222584 zeigen eine Erhöhung der Tumorhypoxie während und nach Verabreichung von PTK787/ZK222584. Interssanterweise unterbindet aber die gleichzeitige Bestrah- 
lung diese Tumorhypoxieerhöhung. Dies könnte erklären, warum die Kombination eine deutlich supraadditive Tumorwachstumsverzögerung bewirkt. Nur eine genaue Erfassung und Analyse der verschiedenen Prozesse in Zusammenhang mit der Blut- und Sauerstoffversorgung im Tumor unter Therapie (Perfusion, Blutvolumen, Sauerstoffpartialdruck, Mikrogefäßdichte etc.) sowie die Etablierung von Surrogatmarkern wird es letztendlich erlauben, die viel versprechende Therapiekombination von Bestrahlung mit Inhibitoren der Angiogenese auch in breit angesetzten klinischen Versuchsreihen auszutesten.

\section{Repopulation als Zielstruktur für die kombinierte Radiochemotherapie - wieder zurück zu allen 4 R der Radiobiologie}

Tumorrepopulation unter Therapie stellt ein großes Problem für die erfolgreiche Tumorkontrolle dar. Insbesondere bei ORL-Karzinomen kann unter Bestrahlung ebenfalls eine akzelerierte Repopulation eintreten, die eine Herausforderung für die Radioonkologie ist. Eine Verkürzung des Therapieschemas ist oftmals nicht möglich, da eine hyperfraktionierte und akzeleriert-fraktionierte $\mathrm{Be}$ strahlung auch mit erheblicher Normalgewebstoxizität einhergehen kann. Aus diesem Grund ist das molekulare Verstehen dieser strahleninduzierten Repopulation von großer Bedeutung, um mit entsprechenden kleinmolekularen Substanzen als Teil einer kombinierten Radiochemotherapie agieren zu können.

In ORL-Karzinomen liegt die EGF-Rezeptortyrosinkinase oftmals mutiert bzw. überexprimiert vor. Auch wenn keine direkte Korrelation zwischen dem EGF-Rezeptorstatus und der TNM-Tumorklassifikation vorliegt, ist das krankheitsfreie Überleben („disease free survival") bei Patienten mit hoher EGF-Rezeptor-Tumorexpression signifikant reduziert. Bezüglich lokoregionaler Tumorkontrolle profitieren v. a. diese Patienten von einem akzelerierten Bestrahlungsschema, was eine direkte Beziehung dieser Tyrosinkinase und akzelerierter Repopulation unter Radiotherapie nahelegt.

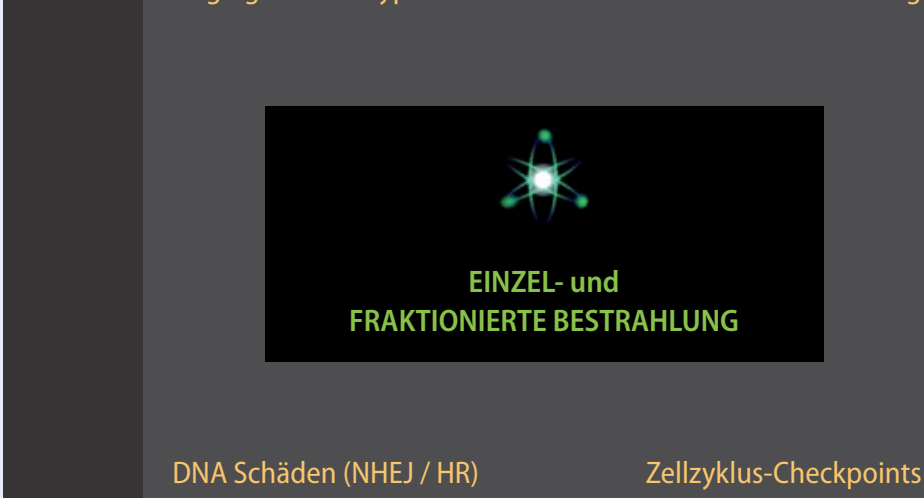

Abb. $2 \Delta$ Radiosensitivität des Tumors. Überblick über die klassischen R der Radiobiologie und deren entsprechende molekulare Zielstrukturen zur Radiosensibilisierung

Interessanterweise induziert die $\mathrm{Be}$ strahlung direkt eine Dimerisierung, Tyrosinphosphorylierung und Aktivierung des EGF-Rezeptors und der damit gekoppelten intrazellulären Signalübermittlungskaskaden. Dies führt zu einem Proliferationsstimulus sowohl von Normalgewebszellen als auch neoplastischer Zellen und kommt somit einer wachstumsfaktorinduzierten, erhöhten Proliferation von überlebenden Tumorzellen nach Bestrahlung gleich.

Diese präklinischen und klinischen Rationalen und Resultate bewirkten, dass die Kombination von EGF-Rezeptorinhibitoren und Bestrahlung intensiv untersucht wurde - mit dem Ziel, die Tumorproliferation durch Inhibierung des EGF-Rezeptors zu reduzieren und durch gleichzeitige zytotoxische Bestrahlung das Tumorvolumen zu kontrollieren. Sowohl präklinische als auch Phase-III-klinische Resultate mit dem Anti-EGFRAntikörper Cetuximab (C225) zeigten eine statistisch signifikante Steigerung einer lokalen Tumorkontrolle und Überleben mit minimal erhöhter Toxizität. Analog dazu zeigt auch die Kombination von Bestrahlung mit EGF-Rezeptortyrosinkinaseinhibitoren eine verbesserte Wachstumskontrolle im präklinischen Rahmen. Interessanterweise kann jedoch nicht gezeigt werden, das die Wirkungsweise dieser kombinierten Radiochemotherapie einzig auf die Kontrolle der Repopulation zurückzuführen ist. Im Gegenteil - präklinische Untersuchungen weisen daraufhin, dass im Bereich der EGF-Rezeptorinhibition in Kombination mit Bestrahlung alle $4 \mathrm{R}$ betroffen sind. Neben der Kontrolle der akzelerierten Repopulation können EGF-Rezeptorinhibitoren ebenfalls die DNA-Reparaturkapazität beeinträchtigen, indem die Kompartimentalisierung der NHEJ-DNA-PKc im Zellkern nach erfolgter DNA-Schädigung verringert wird. Hemmung des EGF-Rezeptors kann aber auch für die Bestrahlung sensibilisieren, indem intrazelluläre Signalübermittlungskaskaden inhibiert (z. B. $\mathrm{PI}_{3} \mathrm{k} /$ Akt-Kaskade) und Schwellwerte reduziert werden (z. B. Apoptose-Schwelle), sodass ein strahleninduzierter Zelltod bereits bei geringerer Dosis eintrifft. Hinzu kommt dass die Hemmung dieses Rezeptors in Tumorzellen ebenfalls eine antiangiogenetische Wirkung hat, indem z. B. weniger VEGF durch die Tumorzelle ausgeschüttet wird $[1,6,8,9,14]$.

Tumorrepopulation ist eine große klinische Herausforderung, welche sowohl durch ein akzeleriertes und hyperfraktioniertes Radiotherapie-Behandlungsschema als auch durch die Kombination mit EGF-Rezeptorinhibitoren kontrolliert werden kann. Eine intensive Forschungstätigkeit wird sich nun der diesbezüglichen Generierung weiterer prädikti- 
ver und prognostischer Faktoren widmen müssen.

\section{Fazit für die Praxis}

\section{Aufgrund einer intrinsischen bzw. erwor- benen Strahlenresistenz während der Karzinogenese oder unter der eigent- lichen Therapie ist die kombinierte Radio- chemotherapie eine attraktive Strategie zur lokalen Tumorkontrolle und krank- heitsfreiem Überleben. Verschiedenste Klassen von Radiosensibilisatoren wur- den während den letzten Jahren entwi- ckelt und v. a. präklinisch ausgetestet. Hier zeigt sich, dass die phänomenolo- gisch sehr gut charakterisierten Bereiche der klassischen Radiobiologie als Leitfa- den sowohl für das Verständnis der mo- lekularen Wirkungsweise als auch für die Entwicklung von Surrogatmarkern die- nen können. Neben der bereits erfolgten Implementierung neuer Bestrahlungs- techniken (z. B. IMRT) wird erwartet, dass sich auch neue Radiochemotherapie- modalitäten mit kleinmolekularen „anti- signaling agents" klinisch erfolgreich durchsetzen werden.}

\section{Korrespondierender Autor}

Prof. Dr. M. Pruschy

Labor für Molekulare Radiobiologie, Klinik für Radio-Onkologie, Universitätsspital Zürich 8091 Zürich

martin.pruschy@usz.ch

Interessenkonflikt. Es besteht kein Interessenkonflikt. Der korrespondierende Autor versichert, dass keine Verbindungen mit einer Firma, deren Produkt in dem Artikel genannt ist, oder einer Firma, die ein Konkurrenzprodukt vertreibt, bestehen. Die Präsentation des Themas ist unabhängig und die Darstellung der Inhalte produktneutral.

\section{Literatur}

1. Ang KK, Berkey BA, Tu X et al. (2002) Impact of epidermal growth factor receptor expression on survival and pattern of relapse in patients with advanced head and neck carcinoma. Cancer Res 62: 7350-7356

2. Bentzen SM (2005) Radiation therapy: intensity modulated, image guided, biologically optimized and evidence based. Radiother Oncol 77: 227-230

3. Bentzen SM (2005) Theragnostic imaging for radiation oncology: dose-painting by numbers. Lancet Oncol 6: 112-117

4. Bernier J, Fuks Z (2004) Advances in translational radiation oncology: from molecular signaling to cancer cure. Int J Radiat Oncol Biol Phys 58: 305 306
5. Bernier J, Hall EJ, Giaccia A (2004) Radiation oncology: a century of achievements. Nat Rev Cancer 4: 737-747

6. Bonner JA, Harari PM, Giralt J et al. (2006) Radiotherapy plus cetuximab for squamous-cell carcinoma of the head and neck. N Engl J Med 354: 567578

7. Brown JM (1999) The hypoxic cell: a target for selective cancer therapy - eighteenth Bruce F. Cain Memorial Award lecture. Cancer Res 59: 58635870

8. Eicheler W, Krause M, Hessel F et al. (2005) Kinetics of EGFR expression during fractionated irradiation varies between different human squamous cell carcinoma lines in nude mice. Radiother Oncol 76: 151-156

9. Eriksen JG, Steiniche T, Overgaard J (2005) The role of epidermal growth factor receptor and E-cadherin for the outcome of reduction in the overall treatment time of radiotherapy of supraglottic larynx squamous cell carcinoma. Acta Oncol 44: 50 58

10. Hall EJ (2000) Radiobiology for the radiologist. Vol. 5th edn. Lippincott Williams and Wilkins

11. Hardcastle IR, Cockcroft X, Curtin NJ et al. (2005) Discovery of potent chromen-4-one inhibitors of the DNA-dependent protein kinase (DNA-PK) using a small-molecule library approach. J Med Chem 48: 7829-7846

12. Hess C, Vuong V, Hegyi I et al. (2001) Effect of VEGF receptor inhibitor PTK787/ZK222584 [correction of ZK222548] combined with ionizing radiation on endothelial cells and tumour growth. Br J Cancer 85: 2010-2016

13. Hofstetter B, Vuong V, Broggini-Tenzer A et al. (2005) Patupilone acts as radiosensitizing agent in multidrug-resistant cancer cells in vitro and in vivo. Clin Cancer Res 11: 1588-1596

14. Huang SM, Harari PM (2000) Modulation of radiation response after epidermal growth factor receptor blockade in squamous cell carcinomas: inhibition of damage repair, cell cycle kinetics, and tumor angiogenesis. Clin Cancer Res 6: 2166-2174

15. Ismail IH, Martensson S, Moshinsky D et al. (2004) SU11752 inhibits the DNA-dependent protein kinase and DNA double-strand break repair resulting in ionizing radiation sensitization. Oncogene 23: 873-882

16. Jackson JR, Gilmartin A, Imburgia C et al. (2000) An indolocarbazole inhibitor of human checkpoint kinase (Chk1) abrogates cell cycle arrest caused by DNA damage. Cancer Res 60: 566-572

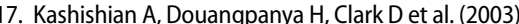
DNA-dependent protein kinase inhibitors as drug candidates for the treatment of cancer. Mol Cancer Ther 2: 1257-1264

18. Kastan MB, Bartek J (2004) Cell-cycle checkpoints and cancer. Nature 432: 316-323

19. Moshous D, Callebaut I, de Chasseval R et al. (2001) Artemis, a novel DNA double-strand break repair/ $\mathrm{V}(\mathrm{D}) \mathrm{J}$ recombination protein, is mutated in human severe combined immune deficiency. Cell 105: 177-186

20. Overgaard J (1989) Sensitization of hypoxic tumour cells - clinical experience. Int J Radiat Bio 56: 801-811

21. Riesterer O, Honer M, Jochum W et al. (2006) Ionizing Radiation Antagonizes Tumor Hypoxia Induced by Anti-Angiogenic Treatment. Submitted

22. Rouse J, Jackson SP (2002) Interfaces between the detection, signaling, and repair of DNA damage. Science 297: 547-551
23. Russell JS, Brady K, Burgan WE et al. (2003) Gleevec-mediated inhibition of Rad51 expression and enhancement of tumor cell radiosensitivity. Cancer Res 63: 7377-7383

24. Wachsberger P, Burd R, Dicker AP (2003) Tumor response to ionizing radiation combined with antiangiogenesis or vascular targeting agents: exploring mechanisms of interaction. Clin Cancer Res 9 : 1957-1971

25. Wang Q, Fan S, Eastman A et al. (1996) UCN-01: a potent abrogator of $\mathrm{G} 2$ checkpoint function in cancer cells with disrupted p53. J Natl Cancer Inst 88: 956-965

26. Westphal CH, Hoyes KP, Canman CE et al. (1998) Loss of atm radiosensitizes multiple $\mathrm{p} 53$ null tissues. Cancer Res 58: 5637-5639

27. Zhou BB, Elledge SJ (2000) The DNA damage response: putting checkpoints in perspective. Nature 408: 433-439 